Proceedings of the Edinburgh Mathematical Society (2005) 48, 143-149 (C)

DOI:10.1017/S0013091503000075 Printed in the United Kingdom

\title{
TIME AVERAGES FOR THE LAPLACE GROUP
}

\author{
DAMIAN C. JACKSON \\ Department of Mathematics and Statistics, Lancaster University, \\ Lancaster LA1 4YF, UK (d.jackson@lancaster.ac.uk)
}

(Received 7 February 2003)

\begin{abstract}
The imaginary powers of the Laplace operator over the circle give a $C_{0}$ group of bounded linear operators on $L_{\theta}^{p}(0,2 \pi)(1<p<\infty)$. Whereas the group is unbounded on $L^{4}$, this paper shows that the $L^{4}$ long-time averages of each $f$ in $L^{2}$ are bounded. This is a Fourier restriction phenomenon.

Keywords: imaginary powers of the Laplace operator; Riesz potentials; Fourier restriction phenomena
\end{abstract}

2000 Mathematics subject classification: Primary 42A15; 42B15

\section{Introduction}

Let $\left\{R^{t}\right\}_{t \in \mathbb{R}}$ be a $C_{0}$ group of bounded linear operators, acting on $L_{\theta}^{p}(0,2 \pi)$ for some $1<p<\infty$. We use $\theta$ to indicate the space variable. Define the long-time average:

$$
A^{(p)} f=\left\{\limsup _{T \rightarrow \infty} \frac{1}{2 T} \int_{-T}^{T}\left\|R^{t} f\right\|_{L_{\theta}^{p}}^{p} \mathrm{~d} t\right\}^{1 / p} .
$$

If the group is bounded with $\left\|R^{t}\right\| \leqslant M$, then clearly it follows that $A^{(p)} f \leqslant M\|f\|_{p}$. Remarkably this inequality holds for some unbounded $C_{0}$ groups. Let $\Delta$ be the Laplace operator over the circle that satisfies $\Delta \mathrm{e}^{\mathrm{i} n \theta}=n^{2} \mathrm{e}^{\mathrm{i} n \theta}$. Zygmund [12, Theorem 1] showed that the periodic Schrödinger group $\mathrm{e}^{\mathrm{i} t \Delta}$ has

$$
\iint_{[0,2 \pi] \times[0,2 \pi]}\left|\mathrm{e}^{\mathrm{i} t \Delta} f(\theta)\right|^{4} \mathrm{~d} t \mathrm{~d} \theta \leqslant 2\|f\|_{L_{\theta}^{2}}^{4} .
$$

We obtain related estimates for the Laplace group $R^{t}=\Delta^{-\mathrm{i} t / 2}$, where

$$
\left(\Delta^{-\mathrm{i} t / 2} f\right)(\theta) \sim \sum_{n \in \mathbb{Z} \backslash\{0\}} \frac{a_{n}}{|n|^{\mathrm{i} i}} \mathrm{e}^{\mathrm{i} n \theta}
$$

for $f=\sum a_{n} \mathrm{e}^{\mathrm{i} n \theta} \in L_{\theta}^{2}$. For brevity we take $a_{0}=0$ throughout. The main result is the following theorem.

Theorem 1.1. Let $f \sim \sum a_{n} \mathrm{e}^{\mathrm{i} n \theta} \in L_{\theta}^{2}$. Then the long-time averages of the Laplace group satisfy

$$
\|f\|_{L_{\theta}^{2}}^{4} \leqslant \lim _{T \rightarrow \infty} \frac{1}{2 T} \int_{-T}^{T}\left\|\Delta^{-\mathrm{i} t / 2} f\right\|_{L_{\theta}^{4}}^{4} \mathrm{~d} t \leqslant 4\|f\|_{L_{\theta}^{2}}^{4} .
$$


This (quite surprising) result relies upon a smoothing effect of the time average, since $\Delta^{-\mathrm{i} t / 2}$ is an unbounded group on $L_{\theta}^{4}$, as we show in $\S \S 2$ and 3 , where we prove $L_{\theta}^{p} \rightarrow L_{\theta}^{p}$ operator bounds for $\Delta^{-\mathrm{i} t / 2}$.

The proof of Theorem 1.1 is carried out for trigonometric polynomials by a combinatorial argument in $\S 4$, and then extended in $\S 5$ to the general $L_{\theta}^{2}$ case. We introduce a Banach space $B_{t}^{4} L_{\theta}^{4}$ of $L_{\theta}^{4}$-valued Bohr almost-periodic functions in time such that $\Delta^{-\mathrm{i} t / 2}$ is bounded as an operator $L_{\theta}^{2} \rightarrow B_{t}^{4} L_{\theta}^{4}$.

The group $\Delta^{-\mathrm{it} / 2}$ arises via the periodic Riesz potential kernel in several applications $[\mathbf{7}, \S 19.3]$. The spectral theory of operator groups on $L_{\theta}^{4}$ is treated in [2].

\section{Upper bounds on the Laplace group}

Proposition 2.1. Let $p>1$ and $r=\max (p, q)$, where $1 / p+1 / q=1$. Then, for $0<\epsilon<2 /(r-2)$, there exist constants $c_{p}(\epsilon), C_{p}(\epsilon)>0$ such that

$$
c_{p}(\epsilon)|t|^{(1 / 2)-(1 / r)} \leqslant\left\|\Delta^{-\mathrm{i} t / 2}\right\|_{L_{\theta}^{p} \rightarrow L_{\theta}^{p}}<C_{p}(\epsilon)(1+|t|)^{1-(2 / r)+\epsilon} \quad(t \in \mathbb{R}) .
$$

In particular, for $p=4$ the following holds:

$$
c_{4}(\epsilon)|t|^{1 / 4} \leqslant\left\|\Delta^{-\mathrm{i} t / 2}\right\|_{L_{\theta}^{4} \rightarrow L_{\theta}^{4}}<C_{4}(\epsilon)(1+|t|)^{(1 / 2)+\epsilon} \quad(t \in \mathbb{R}) .
$$

Proof of the upper bound. The strong form of Marcinkiewicz's Multiplier Theorem $[\mathbf{5}, \S 8]$, applied to $\phi(y)=|y|^{-\mathrm{i} t}$, gives an upper bound for the operator norm. As $\phi$ has constant modulus 1 , and as the variation over dyadic intervals $\left[2^{k}, 2^{k+1}\right]$ is uniformly bounded by $|t|$, this $\phi$ determines an $L_{\theta}^{p}$ multiplier $a_{n} \mapsto \phi(n) a_{n}$ for all $p>1$, and we deduce

$$
\left\|\Delta^{-\mathrm{i} t / 2}\right\|_{L_{\theta}^{p} \rightarrow L_{\theta}^{p}} \leqslant C_{p}(1+|t|) \quad(t \in \mathbb{R}) .
$$

So the operators are bounded, with norms of at most linear growth in $|t|$. Let $r>$ 2 , suppose $0<\epsilon<2 /(r-2)$, and $p=2+(2 / \epsilon)$. Now we may apply Riesz-Thorin interpolation between $L_{\theta}^{2}$ and $L_{\theta}^{p}$. Plugging in the exact value $\left\|\Delta^{-\mathrm{i} t / 2}\right\|_{L_{\theta}^{2} \rightarrow L_{\theta}^{2}}=1$ and the bound (2.3) gives

$$
\left\|\Delta^{-\mathrm{i} t / 2}\right\|_{L_{\theta}^{r} \rightarrow L_{\theta}^{r}}<C_{r}(\epsilon)(1+|t|)^{1-(2 / r)+\epsilon} \quad(t \in \mathbb{R}),
$$

where $\epsilon$ can be made arbitrarily small, at the cost of growth in $C_{r}(\epsilon)$. For $1<q<2$, since $\Delta^{-\mathrm{i} t / 2}$ is self-adjoint, we have

$$
\left\|\Delta^{-\mathrm{i} t / 2}\right\|_{L_{\theta}^{q} \rightarrow L_{\theta}^{q}}<C_{q}(\epsilon)(1+|t|)^{-1+(2 / q)+\epsilon} \quad(t \in \mathbb{R})
$$

by considering the dual exponent $r=q /(q-1)$. Thus the right-hand side of $(2.1)$ is proven.

\section{Lower bound on the Laplace group}

We shall use a test function related to the imaginary part of the periodic zeta function [1] to prove the left-hand inequality of Proposition 2.1. Let $s=\sigma+$ it be within strip 
$\Omega=\{s: 0<\operatorname{Re} s<1\}$. Define

$$
m_{s}(\theta)=\sum_{n=1}^{\infty} \frac{\sin (n \theta)}{n^{1-s}}
$$

If $\sigma<1 / p$, then both $m_{\sigma}$ and $m_{\sigma+\mathrm{i} t}$ are $L_{\theta}^{p}$ functions and the following holds:

$$
m_{s}(\theta)=\left(\Delta^{-\mathrm{i} \tau / 2} m_{s+\mathrm{i} \tau}\right)(\theta) \quad(\tau \in \mathbb{R}) .
$$

The Hurwitz generalized zeta function $[\mathbf{1 0}, \S 2]$ is initially defined by

$$
\zeta(s, a)=\sum_{n=0}^{\infty} \frac{1}{(n+a)^{s}} \quad(a>0)
$$

which is clearly analytic in the half-plane $\operatorname{Re} s>1$ with pole at $s=1$, and may be continued to exponents $s \in \Omega$ via the loop integral

$$
\zeta(s, a)=\frac{\mathrm{e}^{-\mathrm{i} \pi s} \Gamma(1-s)}{2 \pi \mathrm{i}} \int_{C} \frac{z^{s-1} \mathrm{e}^{-a z}}{1-\mathrm{e}^{-z}} \mathrm{~d} z \quad(a>0),
$$

where the path $C$ encircles $\mathbb{R}^{+}$anticlockwise, including only the pole at $z=0$. From the Fourier representation of $\zeta(s, a)$, we derive

$$
m_{s}(\theta)=\frac{\sin \left(\frac{1}{2} \pi s\right) \Gamma(s)}{(2 \pi)^{s}}\left\{\zeta\left(s, \frac{\theta}{2 \pi}\right)-\zeta\left(s, 1-\frac{\theta}{2 \pi}\right)\right\} .
$$

Hence $m_{s}(\theta)$ may be continued to an entire function of $s$. Now making use of the loop integral representation, we obtain

$$
m_{s}(\theta)=\frac{\sin \left(\frac{1}{2} \pi s\right)}{\pi^{s}} K^{s}\left(1-\frac{\theta}{\pi}\right)
$$

where

$$
K^{s}(\beta)=\int_{0}^{\infty} x^{s-1} \frac{\sinh (\beta x)}{\sinh (x)} \mathrm{d} x
$$

and, for $\beta \rightarrow 1^{-}$, we deduce the asymptotic behaviour

$$
K^{s}(\beta) \sim \frac{\Gamma(s)}{(1-\beta)^{s}}=\Gamma(s)\left(\frac{\pi}{\theta}\right)^{s} .
$$

We now consider the approximating integral for $m_{s}(\theta)$, which is a standard Mellin transform [8, p. 521] for $s \in \Omega$ with value

$$
k_{s}(\theta)=\int_{0}^{\infty} \frac{\sin (\theta u)}{u^{1-s}} \mathrm{~d} u=\frac{\sin \left(\frac{1}{2} \pi s\right) \Gamma(s) \operatorname{sgn}(\theta)}{|\theta|^{s}} \quad(-\pi<\theta<\pi) .
$$

Define $\Omega_{p}=\{s: 0<\operatorname{Re} s<1 / p\}$. If $s \in \Omega_{p}$, then $k_{s} \in L_{\theta}^{p}(-\pi, \pi)$, and

$$
\left\|k_{\sigma+\mathrm{i} t}\right\|_{L_{\theta}^{p}}=\frac{\left|\sin \left(\frac{1}{2} \pi(\sigma+\mathrm{i} t)\right) \Gamma(\sigma+\mathrm{i} t)\right|}{(1-p \sigma)^{1 / p} \pi^{\sigma}} .
$$


Given fixed $\sigma \in(0,1)$, as $|t| \rightarrow \infty$ it is well known from Stirling's formula [9, p. 58] that

$$
|\Gamma(\sigma+\mathrm{i} t)| \sim \sqrt{2 \pi} \mathrm{e}^{-(\pi / 2)|t|}|t|^{\sigma-(1 / 2)} .
$$

Thus we have the asymptotic behaviour

$$
\frac{\left\|k_{\sigma}\right\|_{L_{\theta}^{p}}}{\left\|k_{\sigma+\mathrm{i} t}\right\|_{L_{\theta}^{p}}} \sim \frac{\Gamma(\sigma)|t|^{(1 / 2)-\sigma}}{\sqrt{2 \pi}} \quad(|t| \rightarrow \infty)
$$

For large $|t|$, this estimate allows us to obtain the lower bound

$$
\left\|\Delta^{-i t / 2}\right\|_{L_{\theta}^{p} \rightarrow L_{\theta}^{p}} \geqslant \frac{1}{\sqrt{2 \pi}}|t|^{(1 / 2)-(1 / p)} .
$$

\section{Proof of Theorem 1.1 (trigonometric polynomials)}

The left-hand side of Theorem 1.1 follows from Hölder's inequality, as

$$
\|f\|_{L_{\theta}^{2}}=\left\|\Delta^{-\mathrm{i} t / 2} f\right\|_{L_{\theta}^{2}} \leqslant\left\|\Delta^{-\mathrm{i} t / 2} f\right\|_{L_{\theta}^{4}}(t \in \mathbb{R}) .
$$

We now prove the right-hand side for trigonometric polynomials. Let $f=\sum_{-N}^{N} a_{n} \mathrm{e}^{\mathrm{i} n \theta}$ with $a_{0}=0$. The notation $\sum^{N}$ indicates finite sums of this form, and we sum over all indices subject to the stated conditions. Applying the operator $\Delta^{-\mathrm{i} t / 2}$ to $f$ gives

$$
\left|\Delta^{-\mathrm{i} t / 2} f(\theta)\right|^{4}=\left|\sum^{N} \frac{a_{n} \mathrm{e}^{\mathrm{i} n \theta}}{|n|^{\mathrm{i} t}}\right|^{4}=\sum_{n_{1}, n_{2}, n_{3}, n_{4}}^{N} a_{n_{1}} a_{n_{2}} \bar{a}_{n_{3}} \bar{a}_{n_{4}} \mathrm{e}^{\mathrm{i} \theta\left(n_{1}+n_{2}-n_{3}-n_{4}\right)}\left|\frac{n_{3} n_{4}}{n_{1} n_{2}}\right|^{\mathrm{i} t} .
$$

Integrating with respect to $\theta$, we obtain

$$
\left\|\Delta^{-\mathrm{i} t / 2} f\right\|_{L_{\theta}^{4}}^{4}=\int_{0}^{2 \pi}\left|\Delta^{-\mathrm{i} t / 2} f(\theta)\right|^{4} \frac{\mathrm{d} \theta}{2 \pi}=\sum_{n_{1}+n_{2}=n_{3}+n_{4}}^{N} a_{n_{1}} a_{n_{2}} \bar{a}_{n_{3}} \bar{a}_{n_{4}}\left|\frac{n_{3} n_{4}}{n_{1} n_{2}}\right|^{\mathrm{i} t} .
$$

Now we may form the long-time average. Let

$$
S=\lim _{T \rightarrow \infty} \frac{1}{2 T} \int_{-T}^{T} \sum_{n_{1}+n_{2}=n_{3}+n_{4}}^{N} a_{n_{1}} a_{n_{2}} \bar{a}_{n_{3}} \bar{a}_{n_{4}}\left|\frac{n_{3} n_{4}}{n_{1} n_{2}}\right|^{\mathrm{i} t} \mathrm{~d} t .
$$

Terms with $\left|n_{1} n_{2}\right| \neq\left|n_{3} n_{4}\right|$ vanish, hence we arrive at

$$
S=\sum_{\substack{n_{1}+n_{2}=n_{3}+n_{4} \\\left|n_{1} n_{2}\right|=\left|n_{3} n_{4}\right|}}^{N} a_{n_{1}} a_{n_{2}} \bar{a}_{n_{3}} \bar{a}_{n_{4}}
$$

Now separate the case $S^{+}: n_{1} n_{2}=n_{3} n_{4}$ from $S^{-}: n_{1} n_{2}=-n_{3} n_{4}$ to give

$$
S=\sum_{\substack{n_{1}+n_{2}=n_{3}+n_{4} \\ n_{1} n_{2}=n_{3} n_{4}}}^{N} a_{n_{1}} a_{n_{2}} \bar{a}_{n_{3}} \bar{a}_{n_{4}}+\sum_{\substack{n_{1}+n_{2}=n_{3}+n_{4} \\ n_{1} n_{2}=-n_{3} n_{4}}}^{N} a_{n_{1}} a_{n_{2}} \bar{a}_{n_{3}} \bar{a}_{n_{4}}
$$




\section{The circular case}

The $S^{+}$sum reduces to the system

$$
\begin{aligned}
& n_{1}^{2}+n_{2}^{2}=n_{3}^{2}+n_{4}^{2} \\
& n_{1}+n_{2}=n_{3}+n_{4},
\end{aligned}
$$

which corresponds to the intersections of circles and lines at lattice points $\mathbb{Z}^{2}$. This Diophantine system is considered by Zygmund [12], and Bourgain $[\mathbf{4}, \S 2]$, in the context of the Schrödinger group. We may evaluate $S^{+}$precisely; all off-axis points $\left(n_{1}, n_{2}\right)$ on the lattice $\{-N, \ldots, N\} \times\{-N, \ldots, N\}$ give contributions to the sum. Those with $n_{1} \neq n_{2}$ generate two solutions $\left(n_{3}, n_{4}\right)$ and $\left(n_{4}, n_{3}\right)$, whereas those of form $\left(n_{1}, n_{1}\right)$ give just one. Thus we obtain

$$
S^{+}=2\left\{\sum^{N}\left|a_{n_{1}}\right|^{2}\right\}^{2}-\sum^{N}\left|a_{n_{1}}\right|^{4}
$$

and deduce that

$$
\|f\|_{L_{\theta}^{2}}^{4} \leqslant S^{+} \leqslant 2\|f\|_{L_{\theta}^{2}}^{4}
$$

\section{The hyperbolic case}

The other term $S^{-}$is a sum over intersections of hyperbolae and parallel lines

$$
\begin{aligned}
n_{3}+n_{4} & =n_{1}+n_{2} \\
n_{3} n_{4} & =-n_{1} n_{2} .
\end{aligned}
$$

This general Diophantine system may be solved by change of variables. Solutions are less clear than for $S^{+}$. The sum is over a more sparse set, as the only possible solutions are given by

$$
n_{3}, n_{4}=\frac{1}{2}\left(n_{1}+n_{2} \pm \sqrt{\left(n_{1}+3 n_{2}\right)^{2}-8 n_{2}^{2}}\right),
$$

where $n_{3}$ and $n_{4}$ are integers. In general, $S^{-}$is non-empty, for instance $\left(n_{1}, n_{2}, n_{3}, n_{4}\right)=$ $(2,3,6,-1)$ is an element for $N \geqslant 6$. All solutions may be generated using forms reminiscent of Pythagorean triples. Setting $X=n_{1}+3 n_{2}$ and $Y=n_{2}$, we arrive at the following case of Pell's equation:

$$
X^{2}-8 Y^{2}=k^{2}
$$

with $k$ integral. Here we rely on the fact that $\mathbb{Q}(\sqrt{-2})$ is a Euclidean domain [6]. Now assume $(X, Y, k)$ have no pairwise common factor. The only possible common divisor of $X+k, X-k$ is 2 . Thus $X+k=2 P^{2}, X-k=4 Q^{2}$ give the general solution of (4.12) with $(X, Y)$ positive:

$$
(X, Y, k)=\left(P^{2}+2 Q^{2}, P Q, P^{2}-2 Q^{2}\right),
$$

where $P, Q \neq 0$. These give the minimal solutions of $S^{-}$via

$$
\left(n_{1}, n_{2}, n_{3}, n_{4}\right)=\left(X-3 Y, Y, \frac{1}{2}(X-2 Y+k), \frac{1}{2}(X-2 Y-k)\right) .
$$


Putting $x=P-Q$ and $y=Q$ leads to the symmetric form

$$
\left(\begin{array}{c}
n_{1} \\
n_{2} \\
n_{3} \\
n_{4}
\end{array}\right)=\left(\begin{array}{c}
P^{2}+2 Q^{2}-3 P Q \\
P Q \\
P^{2}-P Q \\
2 Q^{2}-P Q
\end{array}\right)=\left(\begin{array}{c}
x(x-y) \\
y(x+y) \\
x(x+y) \\
-y(x-y)
\end{array}\right)
$$

for non-zero $|x| \neq|y|$. These are the basic solutions, which we can scale to give the general solution in the integer lattice to (4.10). We must allow an additional factor $p$, where $p$ is odd and square-free, giving the explicit expansion

$$
S^{-}=8 \operatorname{Re} \sum_{\substack{0<x<y \\ p \in \mathbb{P}}} a_{p\left(x^{2}-x y\right)} a_{p\left(y^{2}+x y\right)} \bar{a}_{p\left(x^{2}+x y\right)} \bar{a}_{p\left(y^{2}-x y\right)},
$$

where $\mathbb{P}=\{ \pm 1, \pm 3, \pm 5, \pm 7, \pm 11, \pm 13, \pm 15, \ldots\}$. The summation is over the valid range of coefficients, that is to say all subscripts must fall inside $\{-N, \ldots,+N\}$, so that $x$ and $y$ must be less than $\sqrt{N / 2 p}$. We can now make the required estimate:

$$
S^{-} \leqslant 2\|f\|_{L_{\theta}^{2}}^{4} \text {. }
$$

Adding this bound to (4.9) gives the right-hand side of Theorem 1.1 for finite sums.

\section{General $L^{2}$ case}

We extend the previous result to the whole of $L_{\theta}^{2}$, making use of the theory of vectorvalued Bohr almost-periodic functions, from $[\mathbf{3}]$ and $[\mathbf{1 1}]$.

Definition 5.1. Let $X$ be a Banach space, and $g: \mathbb{R} \rightarrow X$ be continuous. We say that $\tau \in \mathbb{R}$ is an $\epsilon$-almost period of $g$ if

$$
\|g(t+\tau)-g(t)\|_{X} \leqslant \epsilon \quad(t \in \mathbb{R}) .
$$

The function $g$ is Bohr almost periodic if for each $\epsilon>0$, there exists $\lambda>0$ such that each interval $(t, t+\lambda)$ contains at least one $\epsilon$-almost period $\tau$. Let $B_{t}^{4} X$ be the completion of the space of Bohr almost periodic $X$-valued functions for the norm

$$
\|g\|_{B_{t}^{4} X}^{4}=\lim _{T \rightarrow \infty} \frac{1}{2 T} \int_{-T}^{T}\|g(t)\|_{X}^{4} \mathrm{~d} t .
$$

The Mean Value Theorem for almost periodic functions shows that this limit exists; the Uniqueness Theorem proves that this is indeed a valid norm.

Theorem 5.2. The map $f \mapsto \Delta^{-\mathrm{i} t / 2} f$ is bounded $L_{\theta}^{2} \rightarrow B_{t}^{4} L_{\theta}^{4}$ with norm at most 4 .

Proof. Given $f$ as in Theorem 1.1, let $f_{N}=\sum_{|n|<N} a_{n} \mathrm{e}^{\mathrm{i} n \theta}$, so that, $f_{N} \rightarrow f$ in $L_{\theta}^{2}$, as $N \rightarrow \infty$. Now let $F_{N}(t, \theta)=\sum_{|n|<N} a_{n} \mathrm{e}^{\mathrm{i} n \theta}|n|^{-i t}$. These partial sums are almost periodic in $t$, with values in $L_{\theta}^{4}$, and give a Cauchy sequence $\left(F_{N}\right)$ in $B_{t}^{4} L_{\theta}^{4}$, the Banach space 
obtained by completing the space of finite sums $\sum_{m, n} b_{n} \mathrm{e}^{\mathrm{i} n \theta} r_{m}^{-\mathrm{i} t}$ with respect to the norm (5.2), where $X=L_{\theta}^{4}$. Let $F$ be the limit of this sequence in $B_{t}^{4} L_{\theta}^{4}$. The Fourier coefficients depend continuously on the $B_{t}^{4} L_{\theta}^{4}$ norm, so that we can regard $F$ as a function with

$$
F(t, \theta)=\Delta^{-\mathrm{i} t / 2} f(\theta)
$$

as the interpretation in $L_{\theta}^{2}$ is unambiguous. Since

$$
\left\|F_{N}\right\|_{B_{t}^{4} L_{\theta}^{4}} \rightarrow\|F\|_{B_{t}^{4} L_{\theta}^{4}} \quad \text { and } \quad\left\|f_{N}\right\|_{L_{\theta}^{2}} \rightarrow\|f\|_{L_{\theta}^{2}}
$$

as $N \rightarrow \infty$, we deduce the general theorem from the finite sum case:

$$
\|F\|_{B_{t}^{4} L_{\theta}^{4}}^{4} \leqslant 4\|f\|_{L_{\theta}^{2}}^{4} .
$$

Acknowledgements. While writing this paper the author was supported by an EPSRC research studentship. I thank my supervisor Dr G. Blower for his great patience and guidance.

\section{References}

1. T. M. Apostol, Introduction to analytic number theory (Springer, 1976).

2. E. Berkson, T. A. Gillespie and P. S. Muhly, Abstract spectral decompositions guaranteed by the Hilbert transform, Proc. Lond. Math. Soc. 53 (1986), 489-517.

3. H. Bohr, Almost periodic functions (Chelsea, New York, 1947).

4. J. Bourgain, Fourier transform restriction phenomena for certain lattice subsets and applications to nonlinear evolution equations, Part I, Schrödinger equations, Geom. Funct. Analysis 3 (1993), 107-156.

5. R. E. Edwards AND G. I. Gaudry, Littlewood-Paley and multiplier theory (Springer, 1977).

6. G. H. HARDY AND E. M. WRIGHT, An introduction to the theory of numbers (Clarendon, Oxford, 1979).

7. S. G. Samko, A. A. Kilbas and O. I. Marichev, Fractional integrals and derivatives: theory and applications (Gordon and Breach, London, 1993).

8. I. N. SNEDDON, The use of integral transforms (McGraw-Hill, 1979).

9. E. C. Titchmarsh, The theory of functions, 2nd edn (Oxford University Press, 1939).

10. E. C. Titchmarsh, The theory of the Riemann $\zeta$-function (Oxford University Press, 1951).

11. S. Zaidman, Almost-periodic functions in abstract spaces (Pitman, Boston, MA, 1985).

12. A. ZyGmund, On Fourier coefficients and transforms of functions of two variables, Studia Math. 50 (1974), 189-202. 